\title{
Diagnostic performance of urinary metanephrines for the postmortem diagnosis of hypothermia
}

\author{
Cristian Palmiere • Grzegorz Teresiński • \\ Petr Hejna $\cdot$ Patrice Mangin $\cdot$ Eric Grouzmann
}

Accepted: 6 June 2014/ Published online: 18 July 2014

(C) Springer Science+Business Media New York 2014

\begin{abstract}
The purpose of this study was to assess the diagnostic potential of urinary metanephrines and 3-methoxytyramine compared to urinary catecholamine determination in diagnosing antemortem cold exposure and fatal hypothermia. 83 cases of fatal hypothermia and 144 control cases were included in this study. Catecholamines (adrenaline, noradrenaline and dopamine), metanephrines (metanephrine, normetanephrine) and 3-methoxytyramine were measured in urine collected during autopsy. All tested analytes were significantly higher in hypothermia cases compared to control subjects and displayed a generally satisfying discriminative value, thus indicating urinary catecholamines and their metabolites as reliable markers of cold-related stress and hypothermia related-deaths. Metanephrine and adrenaline had the best discriminative value between hypothermia and control cases compared to other tested analytes, though with different sensitivity and specificity. These can therefore be considered the most suitable markers of cold-related stress.
\end{abstract}

C. Palmiere $(\bowtie) \cdot$ P. Mangin

University Centre of Legal Medicine, Lausanne University

Hospital (CHUV), Rue du Bugnon 21, 1011 Lausanne,

Switzerland

e-mail: cristian.palmiere@chuv.ch

G. Teresiński

Chair and Department of Forensic Medicine, Medical University of Lublin, Lublin, Poland

P. Hejna

Institute of Legal Medicine, Charles University in Prague, Faculty of Medicine in Hradec Králové, University Hospital Hradec, Hradec Kralove, Czech Republic

E. Grouzmann

Service de Biomédecine, Lausanne University Hospital

(CHUV), 1011 Lausanne, Switzerland
Keywords Hypothermia - Catecholamines .

Metanephrines · Urine · Postmortem biochemistry

\section{Introduction}

Catecholamines are major humoral factors and neurotransmitters that increase following various types of acute stress. According to numerous observations, catecholamine levels reflect the activity of both adrenergic neurons and the adrenal medulla, thereby expressing the magnitude of physical stress responses or neuronal dysfunction. Thus, their determination in forensic casework may be suitable in investigating various stress responses involved in the death process in individual cases [1].

The stress caused by exposure to cold is responsible for simpatico-adrenal system activation in both humans and animals. This causes a rise in blood noradrenaline and adrenaline concentrations and their subsequent increases in urine. In hypothermia, catecholamines are produced by the adrenal medulla as well as peripheral sympathicus nerve endings. Catecholamine release from the sympathicoadrenal medullary system has been associated with both metabolic and cardiovascular responses important for heat production and heat loss prevention [2-5].

Urinary catecholamine levels have been reported to increase in hypothermia fatalities. Their measurement is therefore recommended in forensic pathology as routine for the diagnosis of cold-related stress and deaths. Though adrenaline and noradrenaline determination in urine may provide further information to support the diagnosis of death by hypothermia, preservation measures during sample collection and storage were reported to significantly influence catecholamine stability in postmortem specimens. Hence, normal levels of urine catecholamines in 
suspected hypothermia fatalities do not allow this diagnosis to be excluded [6-9].

Observations made in clinical practice have revealed urinary catecholamine degradation in unpreserved samples stored at room temperature with a basic $\mathrm{pH}$. Conversely, special requirements for urinary catecholamine stability during collection and storage at room temperature do not appear necessary to ensure metanephrine, normetanephrine and 3-methoxytyramine stability (the $O$-methylated metabolites of adrenaline, noradrenaline and dopamine, respectively) [10, 11].

In this study, we measured catecholamines, metanephrines and 3-methoxytyramine in the urine samples of hypothermia fatalities and control cases collected during autopsy. The first aim of our study was to assess the diagnostic potential of urinary metanephrines and 3-methoxytyramine compared to classic urinary catecholamines for the diagnosis of antemortem cold exposure. The second aim of our study was to determine whether urinary metanephrines and 3-methoxythyramine are more suitable biochemical markers than urinary catecholamines in the postmortem diagnosis of hypothermia.

\section{Materials and methods}

In order to collect a sufficient number of cases, a study involving 3 medico-legal centers in 3 different European countries was carried out from 2011 to 2013. A hypothermia fatality group consisting of 83 forensic autopsy cases and a control group consisting of 144 medico-legal cases were formed.

Inclusion criteria for the hypothermia group were:

- Circumstantial elements suggesting antemortem cold exposure,

- Autopsy and histology findings indicative of hypothermia (hemorrhagic spots of the gastric mucosa, frost erythema over the extensor surfaces of the large joints, hemorrhages of the synovial membrane and bloody discoloration of synovial fluid, generally observed in about $35-75 \%$ of cases),

- Postmortem biochemical investigation results supporting the diagnosis of hypothermia (noted increases in acetone, beta-hydroxybutyrate, cortisol and free fatty acid concentrations in blood as well as increased free cortisol concentrations in urine),

- Urine availability during autopsy,

- Exclusion of other causes of death based on all postmortem investigation findings.

Control cases were retrospectively selected and included:

- Twenty-four cases of death characterized by a short agonal period. This was defined as a period of a few seconds up to a few minutes ( 8 cases of thoracic gunshot wounds, 8 cases of high falls and 8 cases of motor vehicle crashes). All these cases died without cardiopulmonary resuscitation or hospital admission.

- Forty-eight cases of death characterized by a long agonal period. This was defined as a period lasting from several minutes up to hours ( 8 cases of head trauma, 24 cases of severe thoracic and/or abdominal trauma and 16 cases of alcohol and/or drug intoxication). All these cases died without cardiopulmonary resuscitation or hospital admission.

- Twenty-four cases of sudden cardiac death with acute coronary thrombosis at postmortem examination. All these cases died without cardiopulmonary resuscitation or hospital admission.

- Twenty-four cases of severe thoracic and/or abdominal trauma, who underwent cardiopulmonary resuscitation and hospital admission, with documented catecholamine administration during emergency medical treatment.

- Twenty-four cases of suicide by hanging. All these cases died without cardiopulmonary resuscitation or hospital admission.

Inclusion criteria for the control group were the exclusion of hypothermia as the main or contributory cause of death and the availability of urine during postmortem examination.

Causes of death, demographic data, postmortem intervals (or interval from body discovery), survival times and seasonal distributions pertaining to the studied groups are summarized in Table 1.

Complete conventional autopsy, histology examination of the main organs (hematoxylin-eosin), toxicology, and postmortem biochemical investigations were performed in both the hypothermia and control cases. Autopsies were carried out jointly by two forensic pathologists (at least one board-certified) in accordance with both local standards and international guidelines.

\section{Catecholamine metabolic pathway}

Human plasma contains six readily detectable catechols, compounds containing two adjacent hydroxyl groups on a benzene ring. The main plasma catechols are the three catecholamines (adrenaline, noradrenaline and dopamine), their precursor, L-3,4-dihydroxyphenylalanine (L-DOPA; levodopa), and their deaminated metabolites 3,4-dihydroxyphenylacetic acid (DOPAC) from dopamine and 3,4-dihydroxyphenyl glycol (DHPG, dihydroxyphenylethylene glycol) from noradrenaline.

Catecholamines undergo a complex fate mediated by several enzymes in various combinations. Since these 
Table 1 Summary of the causes of death, demographic data, postmortem intervals (or interval from body discovery), survival times (with short/ long agonal duration, as defined in the text) and seasonal distributions in the studied groups

\begin{tabular}{|c|c|c|c|c|c|}
\hline Cause of death & Gender & Age (range) & Postmortem interval & Survival time & Seasonal distribution \\
\hline Hypothermia (83) & $\begin{array}{l}59 \text { males } \\
24 \text { females }\end{array}$ & $15-81$ & $\begin{array}{l}\text { 4-48 h (from body } \\
\text { discovery) }\end{array}$ & Long agonal period & September-April \\
\hline Gunshot wounds (8) & 8 males & $29-54$ & $5-40 \mathrm{~h}$ & Short agonal period & September-April \\
\hline Fall (8) & $\begin{array}{l}2 \text { males } \\
6 \text { females }\end{array}$ & $49-85$ & $6-30 \mathrm{~h}$ & Short agonal period & September-April \\
\hline Motor vehicle crash (8) & $\begin{array}{l}6 \text { males } \\
2 \text { females }\end{array}$ & $19-53$ & $8-48 \mathrm{~h}$ & Short agonal period & September-April \\
\hline Head trauma (8) & $\begin{array}{l}6 \text { males } \\
2 \text { females }\end{array}$ & $21-72$ & $8-40 \mathrm{~h}$ & Long agonal period & September-April \\
\hline $\begin{array}{l}\text { Thoracic/abdominal } \\
\text { trauma, without CPR (24) }\end{array}$ & $\begin{array}{l}20 \text { males } \\
4 \text { females }\end{array}$ & $23-64$ & $6-48 \mathrm{~h}$ & Long agonal period & September-April \\
\hline $\begin{array}{l}\text { Alcohol/Drug intoxication } \\
\text { (16) }\end{array}$ & 16 males & $38-59$ & $8-40 \mathrm{~h}$ & Long agonal period & September-April \\
\hline $\begin{array}{l}\text { Acute coronary thrombosis } \\
\text { (24) }\end{array}$ & $\begin{array}{l}14 \text { males } \\
10 \text { females }\end{array}$ & $49-66$ & $6-36 \mathrm{~h}$ & - & September-April \\
\hline $\begin{array}{l}\text { Thoracic/abdominal } \\
\text { trauma, with CPR (24) }\end{array}$ & $\begin{array}{l}16 \text { males } \\
8 \text { females }\end{array}$ & $24-74$ & $12-48 \mathrm{~h}$ & Long agonal period & September-April \\
\hline Hanging (24) & $\begin{array}{l}22 \text { males } \\
2 \text { females }\end{array}$ & $27-77$ & $6-48 \mathrm{~h}$ & Short agonal period & September-April \\
\hline
\end{tabular}

Survival times in acute coronary thrombosis deaths were unavailable. CRP: cardiopulmonary resuscitation

enzymes are expressed differently in various tissues, circulating product levels have distinctive sources and reflect specific aspects of sympathetic neuronal and adrenomedullary hormonal system functions.

Catechol- $O$-methyltransferase catalyzes the $O$-methylation of the 3-hydroxyl group of most catechols. 3-methoxytyrosine is the $O$-methylated derivative of L-DOPA, 3-methoxytyramine is mainly that of dopamine, normetanephrine that of noradrenaline and metanephrine that of adrenaline. The corresponding 3-O-methylated metabolite of dopamine (3-methoxytyramine) is not generally considered a metanephrine, a term that refers to metanephrine and normetanephrine.

In most cells, the $O$-methylated compounds that contain amine groups undergo further metabolic breakdown by monoamine oxidase. 3-methoxytyramine deamination yields homovanillic acid (HVA). Normetanephrine and metanephrine deamination yields methoxyhydroxyphenylglycol (MHPG).

In cells that have monoamine-preferring phenolsulfotransferase activity, 3-methoxytyramine, normetanephrine, metanephrine and MHPG undergo extensive sulfate-conjugation. Glucuronides of these compounds may be excreted in bile or, via entry into the circulation, in urine $[12,13]$.
Urine collection and analysis

Urine was collected in all cases by bladder aspiration during postmortem examination $(5-10 \mathrm{ml})$. Collected urine was stored in tubes containing 30-150 $\mu \mathrm{l} \mathrm{HCl} 5 \mathrm{~N}$ to adjust the $\mathrm{pH}$ to around 3. Unpreserved urine samples were also collected for creatinine determination.

Acidified urine samples were stored at $-70{ }^{\circ} \mathrm{C}$ after collection and maintained frozen until analysis. Frozen samples were dispatched in dry ice from each center and transported to the laboratory for analysis. The longest period between freezing and sample analysis was 10 months.

In 20 out of 24 control subjects with severe thoracic and/ or abdominal trauma who had been admitted to hospital, antemortem urine samples $(5-10 \mathrm{ml})$ collected prior to death (4-6 h) were obtained, acidified with $\mathrm{HCl} 5 \mathrm{~N}$ to adjust the $\mathrm{pH}$ to around 3 and stored at $-70{ }^{\circ} \mathrm{C}$ until analysis. These frozen samples were dispatched in dry ice to the laboratory in charge of analysis.

Catecholamines (adrenaline, noradrenaline and dopamine), metanephrines (metanephrine, normetanephrine) and 3-methoxytyramine were analyzed using high-performance liquid chromatography (HPLC) with amperometric detection. The measured values were related to creatinine concentration in urine. Creatinine levels in urine were expressed in $\mathrm{mmol} / \mathrm{l}$. 
Urine creatinine was determined (Jaffé method, rateblanked and compensated) by Roche standard methods on the Roche Modular P system (Roche Diagnostics GmbH, Mannheim, Germany).

Ethics

All cases included in this study underwent medico-legal autopsies as requested by the inquiring authorities. Urine samples are routinely collected during autopsy for toxicological and/or biochemical purposes in all the medico-legal centers involved in this study. Catecholamine and metanephrine measurements were performed as part of medicolegal investigations. All biological samples were anonymized prior to analysis and analyzed in the same laboratory. No ethical approval was necessary to perform biochemical analyses in the collected cases.

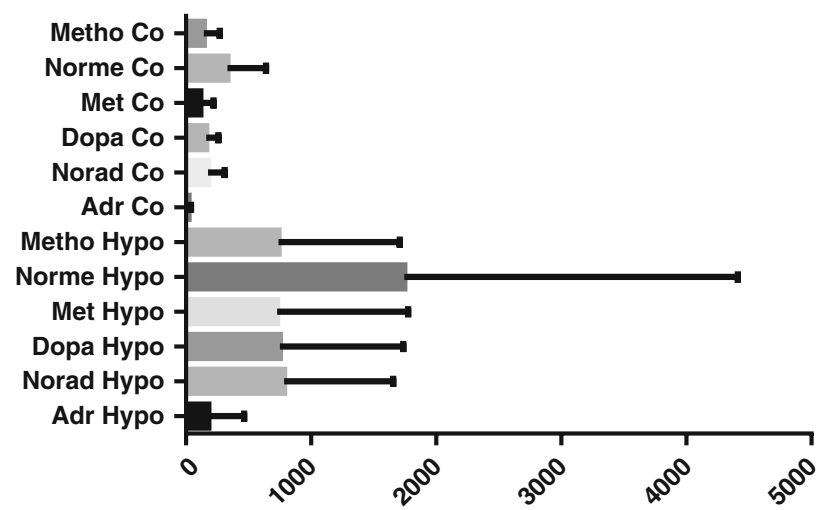

Fig. 1 Graphical representation of the parameter distribution in hypothermia-related deaths (Hypo) and control cases (Co). The concentrations of the measured parameters are expressed in $\mathrm{nmol} / \mathrm{mmol}$ creatinine. Adr adrenaline, Noradr noradrenaline, Dopa dopamine, Met metanephrine, Norme normetanephrine, Metho 3-methoxytyramine
Statistical analysis

Data were analyzed by using the STATA statistical software package (STATA Corporation, College Station, TX, USA). Receiver-operator characteristic (ROC) curves, sensitivity and specificity values were calculated to assess the performance of the selected parameters as diagnostic biomarkers in hypothermia-related deaths. Diagnostic accuracy for the studied parameters was determined by the area under the curve (AUC). Catecholamine, metanephrine and 3-methoxytyramine concentrations in hypothermia and control cases (and within control cases) were compared using nonparametric tests. Statistical significance was set at $p<0.05$. Cutoff levels discriminating hypothermia-related deaths and control cases were not preliminarily identified. Antemortem and postmortem catecholamine, metanephrine and 3-methoxytyramine concentrations were compared using nonparametric tests.

\section{Results}

The graphical representation of the parameter distribution in hypothermia-related deaths and control cases is shown in Fig. 1. Table 2 summarizes ranges, mean values, medians and standard deviations for all tested parameters in both studied groups. Statistical analysis results are reported in Table 3 .

No gender-related or age-dependent differences in the urinary levels of the tested parameters were observed in either group. No correlation between blood ethanol concentration and the urinary values of the measured biomarkers was found in either group.

All tested parameters (adrenaline, noradrenaline, dopamine, metanephrine, normetanephrine and 3-methoxytyramine) were significantly higher in hypothermia-related deaths than in the control group $(p<0.001)$.

Table 2 Summary of the ranges, mean values, medians, and standard deviations for all tested parameters in hypothermia and control cases

\begin{tabular}{|c|c|c|c|c|c|c|}
\hline & Adrenaline & Noradrenaline & Dopamine & Metanephrine & Normetanephrine & Methoxytyramine \\
\hline \multicolumn{7}{|l|}{ Hypothermia } \\
\hline Range & $3-1,523$ & $41-4,336$ & $10-5,523$ & $47-5,891$ & $121-18,756$ & $38-4,650$ \\
\hline Mean value & (178.70) & $(784.30)$ & (750.90) & $(730.30)$ & $(1,745.00)$ & (739.30) \\
\hline Median & (70.00) & (412.00) & $(359.00)$ & $(274.00)$ & (748.00) & $(348.00)$ \\
\hline Standard deviation & $(285.70)$ & $(873.60)$ & $(985.40)$ & $(1,045.00)$ & $(2,666.00)$ & $(969.50)$ \\
\hline \multicolumn{7}{|l|}{ Control } \\
\hline Range & $2-74$ & $8-491$ & $67-500$ & $8-437$ & $62-1,092$ & $25-411$ \\
\hline Mean value & (21.68) & $(171.50)$ & (159.60) & $(114.60)$ & $(337.50)$ & (144.80) \\
\hline Median & (14.00) & $(143.00)$ & (120.00) & (82.00) & $(218.00)$ & $(100.00)$ \\
\hline Standard deviation & (18.10) & (131.30) & (95.65) & (106.50) & $(306.30)$ & (125.80) \\
\hline
\end{tabular}

Urine concentrations are expressed in $\mathrm{nmol} / \mathrm{mmol}$ creatinine 
Table 3 Summary of statistical analysis results for all tested parameters (the value that provided the best pairing of sensitivity and specificity, specificity, sensitivity, true positives, true negatives, false positives, false negatives and AUC). Data have been calculated based on the value that provided the best pairing of sensitivity and specificity

\begin{tabular}{|c|c|c|c|c|c|c|c|}
\hline & Adrenaline & Noradrenaline & Dopamine & Metanephrine & Normetanephrine & Methoxytyramine & $\mathrm{A} / \mathrm{N}$ \\
\hline Cutoff value & 40 & 493 & 251 & 95 & 280 & 126 & 0.192 \\
\hline Sensitivity & $(87 \%)$ & $(100 \%)$ & $(90 \%)$ & $(72 \%)$ & $(72 \%)$ & $(75 \%)$ & $(73 \%)$ \\
\hline Specificity & $(72 \%)$ & $(48 \%)$ & $(65 \%)$ & $(87 \%)$ & $(81 \%)$ & $(78 \%)$ & $(53 \%)$ \\
\hline True positive & $(60)$ & $(40)$ & $(54)$ & $(72)$ & $(67)$ & $(65)$ & (44) \\
\hline True negative & $(52)$ & $(60)$ & $(54)$ & $(43)$ & (43) & $(45)$ & (44) \\
\hline False positive & (8) & $(0)$ & (6) & (17) & (17) & $(15)$ & (16) \\
\hline False negative & $(23)$ & $(43)$ & (29) & (11) & (16) & (18) & (39) \\
\hline AUC & $(0.8395)$ & $(0.7944)$ & $(0.8182)$ & $(0.8543)$ & $(0.81834)$ & $(0.8105)$ & $(0.6159)$ \\
\hline $95 \%$ specificity value & 71 & & & 354 & & & \\
\hline Sensitivity & $(49 \%)$ & & & $(43 \%)$ & & & \\
\hline Specificity & $(95 \%)$ & & & $(95 \%)$ & & & \\
\hline True positive & (41) & & & (36) & & & \\
\hline True negative & (57) & & & $(57)$ & & & \\
\hline False positive & (3) & & & (3) & & & \\
\hline False negative & (42) & & & $(47)$ & & & \\
\hline
\end{tabular}

Cutoff values are expressed in $\mathrm{nmol} / \mathrm{mmol}$ creatinine

The value providing the best specificity $(95 \%)$ was also indicated for adrenaline and metanephrine. Based on these values, sensitivity, true positives, true negatives, false positives and false negatives were also calculated

The values that provided the best pairing of sensitivity and specificity were chosen as cutoff values for each analyzed parameter. Using a cutoff value of $95 \mathrm{nmol} / \mathrm{mmol}$ creatinine, metanephrine determination allowed 72 out of 83 hypothermia cases $(87 \%)$ to be identified (sensitivity $72 \%)$. Interestingly, using a cutoff value of $40 \mathrm{nmol} / \mathrm{mmol}$ creatinine, adrenaline determination allowed 52 out of 60 control cases ( $87 \%$ ) to be identified (specificity $72 \%$ ).

Metanephrine showed the best discriminative power in differentiating between hypothermia and control cases (AUC 0.8543), followed by adrenaline (AUC 0.8395). However, all tested parameters, individually considered, showed satisfying discriminative values, the least discriminating being noradrenaline (AUC 0.7944). The adrenaline-to-noradrenaline ratio had the least satisfying diagnostic performance (AUC 0.6159).

In the control group, absolute values for adrenaline, noradrenaline, dopamine, metanephrine, normetanephrine and 3-methoxytyramine displayed no significant differences relating to the type of death and length of the agonal period. Thoracic and/or abdominal trauma cases with catecholamine administration during emergency medical treatment had the highest concentrations of all tested parameters, though still significantly lower than those found in hypothermia-related deaths.

Comparisons between antemortem and postmortem urine catecholamine, metanephrine and 3-methoxytyramine concentrations in 20 out of 24 control subjects with severe thoracic and/or abdominal trauma who had been admitted to hospital prior to death revealed lower levels in postmortem samples, even though differences between antemortem and postmortem specimens were not statistically significant. This would suggest that measurements of urine catecholamine, metanephrine and 3-methoxytyramine in postmortem samples collected up to $48 \mathrm{~h}$ after death might provide reliable results.

\section{Discussion}

Despite promising advances in several fields of forensic research in recent years, the postmortem diagnosis of fatal hypothermia remains challenging. Whereas the absence of supportive macroscopic, microscopic and biochemical findings does not allow the diagnosis to be ruled out, their presence does not necessarily indicate it. Indeed, macroscopic and microscopic findings as well as biochemical results may vary considerably from case to case and be influenced by numerous factors and circumstances, thus making them only a possible indication of hypothermia. Biochemical investigations are useful in detecting adaptation responses to stress and metabolic changes occurring after exposure to cold. Nevertheless, abnormalities in biochemical results must be interpreted carefully as they do not allow the diagnosis to be either categorically excluded or formally confirmed [5-9, 14-18]. 
Table 4 Summary of the main results reported by various research teams focusing on postmortem measurements of catecholamines in hypothermia and control cases

\begin{tabular}{|c|c|c|c|}
\hline Authors & Analyzed molecule(s) & Analyzed fluid(s) & Main result(s) \\
\hline Hirvonen et al. $[19,20]$ & $\begin{array}{r}\text { Adrenaline and } \\
\text { noradrenaline }\end{array}$ & Urine & $\begin{array}{l}\text { Increased total urinary catecholamine levels } \\
\text { in hypothermia. Increases in adrenaline } \\
\text { levels proportionally more important than } \\
\text { in noradrenaline }\end{array}$ \\
\hline Sadler and Pounder [23] & $\begin{array}{l}\text { Adrenaline, noradrenaline } \\
\text { and dopamine }\end{array}$ & Urine & $\begin{array}{l}\text { High catecholamine levels in hypothermia. } \\
\text { Adrenaline-to-noradrenaline ratio higher } \\
\text { than } 1 \text { in hypothermia cases: not confirmed }\end{array}$ \\
\hline Tormey et al. [22] & $\begin{array}{l}\text { Adrenaline, noradrenaline } \\
\text { and dopamine }\end{array}$ & Urine & $\begin{array}{l}\text { Little changes in urine catecholamine stored } \\
\text { at room temperature for } 3 \text { days ( } \mathrm{pH} \text { range } \\
0.5-7.0)\end{array}$ \\
\hline Wilke et al. [25] & $\begin{array}{r}\text { Adrenaline and } \\
\text { noradrenaline }\end{array}$ & $\begin{array}{l}\text { Heart blood, femoral blood, } \\
\text { vitreous humor, urine, liquor }\end{array}$ & $\begin{array}{l}\text { Adrenaline-to-noradrenaline ratio higher than } \\
1 \text { in urine in hypothermia cases: not } \\
\text { confirmed }\end{array}$ \\
\hline Zhu et al. [24] & $\begin{array}{l}\text { Adrenaline, noradrenaline } \\
\text { and dopamine }\end{array}$ & $\begin{array}{l}\text { Postmortem serum (peripheral } \\
\text { and cardiac blood) }\end{array}$ & $\begin{array}{l}\text { Low catecholamine levels in both peripheral } \\
\text { and cardiac blood in hypothermia }\end{array}$ \\
\hline Ishikawa et al. [21] & $\begin{array}{l}\text { Adrenaline, noradrenaline } \\
\text { and dopamine }\end{array}$ & $\begin{array}{l}\text { Postmortem serum (cardiac } \\
\text { blood) and cerebrospinal } \\
\text { fluid }\end{array}$ & $\begin{array}{l}\text { Low catecholamine levels in both cardiac } \\
\text { blood and cerebrospinal fluid in } \\
\text { hypothermia }\end{array}$ \\
\hline Pakanen et al. [9] & $\begin{array}{l}\text { Adrenaline and } \\
\text { noradrenaline }\end{array}$ & Urine & $\begin{array}{l}\text { Adrenaline values proportionally more } \\
\text { elevated than noradrenaline values in } \\
\text { hypothermia. Adrenaline-to-noradrenaline } \\
\text { ratio }>1 \text { valuable in detecting antemortem } \\
\text { cold stress }\end{array}$ \\
\hline Palmiere et al. [7] & $\begin{array}{r}\text { Adrenaline and } \\
\text { noradrenaline }\end{array}$ & Urine & $\begin{array}{l}\text { Adrenaline mean value in hypothermia: } \\
167 \mathrm{nmol} / \mathrm{mmol} \text { creatinine } \\
\text { Adrenaline mean value in controls: } 29 \mathrm{nmol} / \\
\text { mmol creatinine }\end{array}$ \\
\hline Ishikawa et al. [1] & $\begin{array}{l}\text { Adrenaline, noradrenaline } \\
\text { and dopamine }\end{array}$ & $\begin{array}{l}\text { Right heart blood, pericardial } \\
\text { and cerebrospinal fluids }\end{array}$ & $\begin{array}{l}\text { Low adrenaline and noradrenaline levels in } \\
\text { pericardial and cerebrospinal fluids in } \\
\text { hypothermia }\end{array}$ \\
\hline
\end{tabular}

In the forensic setting, investigations by Hirvonen et al. undoubtedly made a great contribution to the understanding of catecholamine behavior following cold exposure [2$4,19,20]$. These authors evaluated plasma and urine catecholamine levels in animals exposed to cold, patients undergoing cardiac surgery and individuals who died of hypothermia. The results of these studies repeatedly confirmed that urine catecholamines were increased in hypothermia deaths. According to Hirvonen et al. [20], urine adrenaline levels were proportionally higher than noradrenaline concentrations. Based on these findings, the authors suggested that a catecholamine index (adrenalineto-noradrenaline ratio) greater than 1 , instead of the individually considered increases in urine adrenaline and noradrenaline concentrations, was more valuable in indicating antemortem exposure to cold and subsequent stress responses. The same conclusions as above were reached more recently by Pakanen et al. [9].

Numerous research teams have focused on catecholamine measurements in urine, blood and other biological fluids collected during autopsy, especially in relation to the cause of death and duration of the agonal period (Table 4). In general terms, the results of these investigations confirmed the usefulness of catecholamine determination in urine for the postmortem diagnosis of hypothermia. On the other hand, the importance of the adrenaline-to-noradrenaline ratio in corroborating the hypothesis of death due to cold exposure was put into question in some cases $[1,7$, 9, 19-25].

In our case series, the adrenaline-to-noradrenaline ratio showed the least satisfying diagnostic performance in comparison to other tested parameters and did not provide any additional diagnostic information compared to urinary adrenaline and metanephrine levels individually considered.

To the extent of our knowledge, no research has specifically explored postmortem catecholamine stability in human urine. Conversely, numerous clinical studies have investigated catecholamine stability in plasma and urine under various storage conditions, temperatures and $\mathrm{pHs}$. Some of these studies have also assessed metanephrine stability in preserved, unpreserved and frozen urine samples $[10,11,26,27]$. 
Clinical investigations have revealed that catecholamine concentrations in urine do not decrease in frozen samples, either acidified or unpreserved. Immediate freezing prevents significant free amine degradation or sulfoconjugates deconjugation. Immediate acidification limits catecholamine degradation whereas acidification after $24 \mathrm{~h}$ prevents further decay. Even though special requirements during collection and storage at room temperature do not appear necessary for metanephrines, at least within 1 week of storage, immediate acidification to $\mathrm{pH} 4$ seems to be a general safety precaution to prevent degradation. Immediate freezing or immediate acidification after collection and subsequent freezing should therefore be recommended to avoid catecholamine and metanephrine decay [10, 11, 26, 27].

The results of our investigations appear to be in agreement with the conclusions of the aforementioned clinical studies and confirm that urine acidification and immediate freezing post collection can be considered general safety measures recommended to prevent molecule degradation. In addition, our results seem to indicate that catecholamines, metanephrines and 3-methoxytyramine in acidified urine stored at $-70{ }^{\circ} \mathrm{C}$ may prove stable for up to several months. Suggested measures for storage in the forensic setting include utmost prompt urine collection and acidification to $\mathrm{pH}$ 3-4 immediately post collection (prior to or during postmortem examination). Nevertheless, as suggested by the results of clinical observations, urine acidification is not absolutely mandatory (neither for catecholamines nor metanephrine and 3-methoxythyramine measurements) if samples are immediately frozen post collection, unless there is evidence of alkalosis before death.

In the study presented herein, all tested parameters were significantly higher in hypothermia cases compared to control subjects and displayed a generally satisfying discriminative value. Adrenaline provided better diagnostic performance than noradrenaline and dopamine, as did metanephrine compared to normetanephrine and 3-methoxythyramine. These findings confirm that urinary catecholamines (and their metabolites) could be reliable markers of cold-related stress and that increases in these markers might thus corroborate the hypothesis of hypothermia related-deaths.

Based on our findings, we cannot conclude that urinary metanephrine, normetanephrine and 3-methoxythyramine are more accurate biomarkers than urinary catecholamines for the identification of stress responses following antemortem cold exposure. Nevertheless, we can affirm that increased adrenaline and metanephrine concentrations in urine may support the diagnosis of antemortem cold exposure and hypothermia related-deaths to a greater extent than the other tested molecules.

Lastly, in our case series, no correlation between blood ethanol concentrations and the urinary values of measured biomarkers was observed. These results are in agreement with those reported by Pakanen et al. [9], who found similar ethanol concentrations in hypothermia cases with both normal and increased catecholamine concentrations. Conversely, they do not seem to confirm the conclusions of the studies performed by Hirvonen et al. [19, 20], who had suggested an inverse relationship between urine catecholamine levels and blood ethanol concentrations.

\section{Key points}

1. Urinary catecholamines and their metabolites can be considered reliable markers of stress responses related to cold exposure.

2. Increases in urine adrenaline and metanephrine concentrations may support the diagnosis of antemortem cold exposure and hypothermia related-deaths.

3. Urine samples collected after death for catecholamine and metanephrine measurement should be frozen as soon as possible.

4. The simultaneous detection of various biochemical markers is the best way to formulate correct conclusions pertaining to antemortem cold exposure and death by hypothermia.

\section{References}

1. Ishikawa T, Quan L, Michiue T, Kawamoto O, Wang Q, Chen $\mathrm{JH}$, Zhu BL, Maeda H. Postmortem catecholamine levels in pericardial and cerebrospinal fluids with regard to the cause of death in medicolegal autopsy. Forensic Sci Int. 2013;228: $52-60$.

2. Hanhela R, Hollmen A, Huttunen P, Hirvonen J. Plasma catecholamines, corticosterone, glucose and fatty acids concentrations and mean arterial pressure and body temperature in haemorrhagic hypovolaemia, hypothermia and a combination of these in the rabbit. Acta Physiol Scand. 1990;139:441-9.

3. Hirvonen J, Huttunen P. Hypothermia markers: serum, urine and adrenal gland catecholamines in hypothermic rats given ethanol. Forensic Sci Int. 1995;72:125-33.

4. Lapinlampi TO, Hirvonen JI. Catecholamines in the vitreous fluid and urine of guinea pigs dying of cold and the effect of postmortem freezing and autolysis. J Forensic Sci. 1986;31:1357-65.

5. Bańka K, Teresiński G, Buszewicz G. Free fatty acids as markers of death from hypothermia. Forensic Sci Int. 2014;234:79-85.

6. Palmiere C, Mangin P. Postmortem biochemical investigations in hypothermia fatalities. Int J Legal Med. 2013;127:267-76.

7. Palmiere C, Bardy D, Letovanec I, Mangin P, Augsburger M, Ventura F, Iglesias K, Werner D. Biochemical markers of fatal hypothermia. Forensic Sci Int. 2013;226:54-61.

8. Palmiere C, Teresiński G, Hejna P. Postmortem diagnosis of hypothermia. Int J Legal Med. 2014;128(4):607-14.

9. Pakanen L, Kortelainen ML, Särkioja T, Porvari K. Increased adrenaline to noradrenaline ratio is a superior indicator of antemortem hypothermia compared with separate catecholamine concentrations. J Forensic Sci. 2011;56:1213-8. 
10. Willemsen JJ, Ross HA, Lenders JW, Sweep FC. Stability of urinary fractionated metanephrines and catecholamines during collection, shipment, and storage of samples. Clin Chem. 2007; 53:268-72.

11. Roberts NB, Higgins G, Sargazi M. A study on the stability of urinary free catecholamines and free methyl-derivatives at different $\mathrm{pH}$, temperature and time of storage. Clin Chem Lab Med. 2010;48:81-7.

12. Oeltmann T, Carson R, Shannon JR, Ketch T, Robertson D. Assessment of $O$-methylated catecholamine levels in plasma and urine for diagnosis of autonomic disorders. Auton Neurosci. 2004;116:1-10

13. Goldstein DS, Eisenhofer G, Kopin IJ. Sources and significance of plasma levels of catechols and their metabolites in humans. J Pharmacol Exp Ther. 2003;305:800-11.

14. Zhou C, Bright F, Winskog C, Yool AJ, Byard RW. Lethal hypothermia in an animal model, not associated with basal renal epithelial vacuolization. J Forensic Leg Med. 2014;21:14-6.

15. Bright FM, Winskog C, Tsokos M, Walker M, Byard RW. Issues in the diagnosis of hypothermia: a comparison of two geographically separate populations. J Forensic Leg Med. 2014;22: 30-2.

16. Bright F, Winskog C, Walker M, Byard RW. Why are Wischnewski spots not always present in lethal hypothermia? The results of testing a stress-reduced animal model. J Forensic Leg Med. 2013;20:785-7.

17. Bańka K, Teresiński G, Buszewicz G, Mądro R. Glucocorticosteroids as markers of death from hypothermia. Forensic Sci Int. 2013;229:60-5.

18. Bright F, Gilbert JD, Winskog C, Byard RW. Additional risk factors for lethal hypothermia. J Forensic Leg Med. 2013;20: 595-7.
19. Hirvonen J. Necropsy findings in fatal hypothermia cases. Forensic Sci. 1976;8:155-64.

20. Hirvonen J, Huttunen P. Increased urinary concentration of catecholamines in hypothermia deaths. J Forensic Sci. 1982;27: 264-71.

21. Ishikawa T, Yoshida C, Michiue T, Perdekamp MG, Pollak S, Maeda H. Immunohistochemistry of catecholamines in the hypothalamic-pituitary-adrenal system with special regard to fatal hypothermia and hyperthermia. Leg Med (Tokyo). 2010;12: $121-7$.

22. Tormey WP, Carney M, FitzGerald RJ. Catecholamines in urine after death. Forensic Sci Int. 1999;103:67-71.

23. Sadler DW, Pounder DJ. Urinary catecholamines as markers of hypothermia. Forensic Sci Int. 1995;76:227-30.

24. Zhu BL, Ishikawa T, Michiue T, Li DR, Zhao D, Quan L, Oritani S, Bessho Y, Maeda H. Postmortem serum catecholamine levels in relation to the cause of death. Forensic Sci Int. 2007;173: 122-9.

25. Wilke N, Janssen H, Fahrenhorst C, Hecker H, Manns MP, Brabant EG, Tröger HD, Breitmeier D. Postmortem determination of concentrations of stress hormones in various body fluidsis there a dependency between adrenaline/noradrenaline quotient, cause of death and agony time? Int J Legal Med. 2007;121: 385-94.

26. Chan EC, Wee PY, Ho PC. Evaluation of degradation of urinary catecholamines and metanephrines and deconjugation of their sulfoconjugates using stability-indicating reversed-phase ion-pair HPLC with electrochemical detection. J Pharm Biomed Anal. 2000;22:515-26.

27. Miki K, Sudo A. Effect of urine $\mathrm{pH}$, storage time, and temperature on stability of catecholamines, cortisol, and creatinine. Clin Chem. 1998;44:1759-62. 